\title{
Scientific Research Project Oriented Intelligence Research Knowledge Push Model Building
}

\author{
Zhi-Ping GONG, Yang-Qi ZHANG \\ ${ }^{1}$ Military Transportation Institute of Military Commission Logistics Support Department Tianjin, China \\ ${ }^{2}$ Military Transportation Institute of Military Commission Logistics Support Department Tianjin, China \\ gy656694@163.com,zhangyangqi@gmail.com
}

Keywords- Intelligence research, knowledge push and model building

\begin{abstract}
Scientific research project oriented intelligence research pays stress on the mutual assistance between the researchers of both science and intelligence and thence the push service is of great significance in knowledge expression. This article, starting with the issues existing in the service of the intelligence research for scientific research project, will expound the model building for the scientific research project oriented intelligence research knowledge push.

Intelligence research is a process by intelligence agents who formulate research topics according to clients' requirements, pertinently collecting, analyzing, concentrating information and reporting. A qualified intelligence research report not only stands for the capability of the researchers but also contains a mass of valuable information for the reference of the scientific researches.
\end{abstract}

\section{Issues Existing in the Service of Intelligence Research for Scientific Research Projects}

\section{Untight Coalescence of Intelligence Research and Scientific Research Projects}

Normally, the targeted topics of the intelligence research are raised by the agents according to their department's plans, key projects or inquiries to correlated scientific researchers, but the traditional independent research methods of isolation between intelligence and scientific research determine that the intelligence agents are hard to blend themselves in the scientific research and know fewer and less about the needs of the research. Due to the limit of their own knowledge background, working environment and ability and quality, the agents are less likely to raise the research topics fitting and well-meeting the stringent needs of the scientific researchers [1].

\section{Long Period and Weak Timeliness of Intelligence Research}

It is always that the routine of intelligence research is to plan, apply for and assess the topic and determine the schedule (normally 1 year for a general project and 2 years for a key project), release the plan and carry out the procedure of the project according to the plan in the beginning of a year. Roughly speaking, the period of one or two years for a project is not too long, but for scientific researchers, in most cases, a specific problem or a key link required to be solved indicates that the intelligence is of provisionality and urgency and far more pressing than an annual report. The information would be far less functional for scientific guidance and less valuable for reference if it was out of date.

\section{Deficient Information Source and Insufficient Database}

As some of the scientific researches relating to the state defense and military industry, the valuable information from the Internet is quite rare and still submerged in the ocean of messages. The problem is that to extract the useful information from the Internet is never easier than to look for the needle in a bundle of hay, because the data may only flow out from very few of the specialized websites with limited, slowly updated and non-vital information. In the meanwhile, the slow-going digitalization of the 
documents, historical reports and technical magazines, relatively laggard specialized database, the imperfect and un-updated information because of weak technical strength, labor shortage and tight budgets cannot provide easy and convenient query system and may further loose the re-use of the possessed documents.

\section{The Way to Serve Better Scientific Research Project}

\section{An Accurate Topic Shall be Selected}

Topic selection is crucial and critical to intelligence research and it is also an examination for the capability of intelligence agents. The selection is mainly based on the decision made by the higher ranked officers and the consideration of the needs of scientific research projects. However, the pertinence of the research topic is more important. The project must be initiated and arranged in consideration of the requirements of the scientific research on its long and short terms and potentiality, aiming at key and hot issues and focusing on the service construction and development. It is triable to set some scientific research project as a "customer" and emphasize the sense of customer-service to obtain guidance and support on the directions and contents of intelligence research from related scientific researchers without detours. Besides, it is necessary to enhance the communication between intelligence and scientific researchers to let the former better know the needs of the latter and adjust timely the research topic.

\section{Quick Response}

Improve the way of revealing intelligence research achievement, change the traditional year-end reporting into dynamic reporting (monthly or quarterly) providing the scientific researchers with brief and periodic reports on key data collected and studied, take the feedback from the "customer" for the adjustment of the research on directions, way of information supplying and frequency, and give a final, overall and comprehensive report when the research is completed. This will shorten the period from information collection to application, meet the temporary and timely needs of scientific researchers, give as well a better understand of the scientific research project to the intelligence agents and increase the researching efficiency.

As a qualified intelligence agent, it is necessary to have good knowledge about the professional fields besides the basic skills of research. On one hand, an intelligence agent must study the special knowledge integrating with the needs of job. On the other hand, the intelligence agent must go to the research frontier to communicate and interact with the scientific researchers to know at first hand the actual needs of the researchers and scientific research project. With close relationship and complete communication with the scientific researchers, the intelligence agent can purposely collect and accumulate the information in advance and provide advanced and speedy service to have the scientific research play the role of guiding and of pioneering and informing in intelligence research[2].

\section{New Methods}

Based on the traditional qualitative research method, weighing desirability and possibility, the intelligence research shall select and apply a suitable method with increased ratio of quantitative and semi-quantitative ways and in the combination of qualitative and quantitative means to give out wellfounded and convincing views and suggestions. And also for the reveal of the research achievements, instead of the pure text report, it is necessary to explore the possibility of multimedia applications to give a brand new, distinctive and intuitive report with texts, pictures, sounds and videos that will raise the impact and acceptability of the achievements of the intelligence research. 


\section{Highly Qualified}

The quality and capability of the intelligence agents are the basic assurance of intelligence research and the capabilities of hunting for information and studying information are essential skills for an intelligence agent. Hunting for information is to subtly capture, screen, concentrate, process and recreate the valuable data, in a very short period, in the oceans of complicated information and on some insignificant blind sides with all kinds of search engines and tools and by different means and sources. This capability is the foundation of intelligence research and the timeliness, effectiveness and scientificity of information capture will directly influence the quality of the research results.

Studying information refers to the capability of an intelligence agent to collate, deduce, and analyze the information captured by qualitative and quantitative means according to the present situation in China and the capability of offering advisory advice. This is the core and key of the integrative competence for intelligence agents [3].

\section{Scientific Research Project Oriented Intelligence Research Knowledge Push Model Building}

\section{Model Building}

Scientific research oriented intelligence research means to have the intelligence agents be infused into the whole process of scientific research project. Under the impact of the safeguard mechanism, intelligence research will be embedded in the project to sense the needs of scientific research and provide specialized and individualized knowledge pushing service. While emphasising the orientation of scientific project in this article, the model based on embedding style knowledge push service will be structured into the service provider, service receiver, service content and service environment [4]. (See Chart 1)

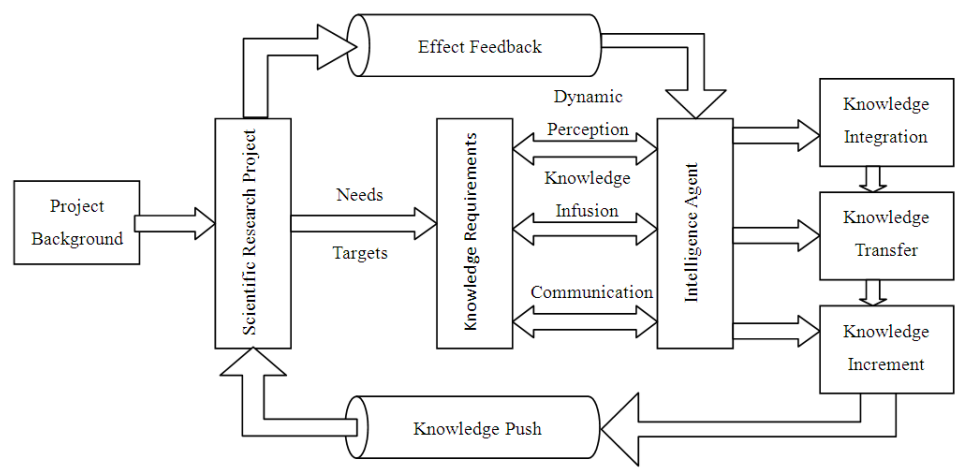

Fig.1.Scientific research project oriented intelligence research Knowledge push model

\section{The Service Provider and Receiver}

The service provider refers to the intelligence agent who impels and executes the knowledge service. The service receiver is the scientific research personnel. By its own knowledge and skills, the service provider will provide, in the embedding style, the receiver with the required knowledge service upon the needs of the project at the receiver.

Both the service provider and receiver have the same prospect. By timely communication and feedback, the requirements are effectively expressed and sensed and the knowledge service can be provided and received with high efficiency [5].

\section{Service Content}

Service content is the final achievements (i.e. intellectual products) of the provider with its perception of the requirement of the receiver and its data analysis and excavation. The intellectual product is the 
gem of wisdom. It is the carrier of the knowledge transfer from the service provider to the receiver and the knowledge processed by the provider with its own knowledge and skills and directly determines the services effects and quality [6].

\section{Service Environment}

The service environment is that of scientific research in which both the provider and receiver carry forward the knowledge service, which includes two aspects---guarantee mechanism and service circumstances.

A. Guarantee mechanism is a guarantee incentive system in which both the service provider and the receiver conduct a long term interaction oriented by a fixed scientific research project. This system will directly influence the service willingness of service provider and feedback willingness of service receiver.

B. Service circumstances mean the relation between the provider and the receiver in different service approaches, which exert the degrees of trust and reciprocity, disparities between organizations and professions.

The degree of trust is the mutual fiduciary relation, which will influence the width and depth of the service; the degree of reciprocity is the beneficial expectation of either party, which will affect the enthusiasm of interaction; organizational disparity is the similarity of the organizations in culture, which will show the smoothness of interaction; professional disparity is the similarity of the organizations in professional background, which will influence the cognitive structure and capability.

\section{Service Effect Model}

The knowledge skill and the capability of knowledge process and output of an intelligence agent as well as the capability of knowledge absorbing and innovation of a researcher will play an important role in the service effects [7].

Oriented by the scientific research project and centered on the knowledge requirements of the service receiver, the service provider has a stronger power of knowledge processing and integration. There is some disparity between the provider and the receiver. (See Chart 2)

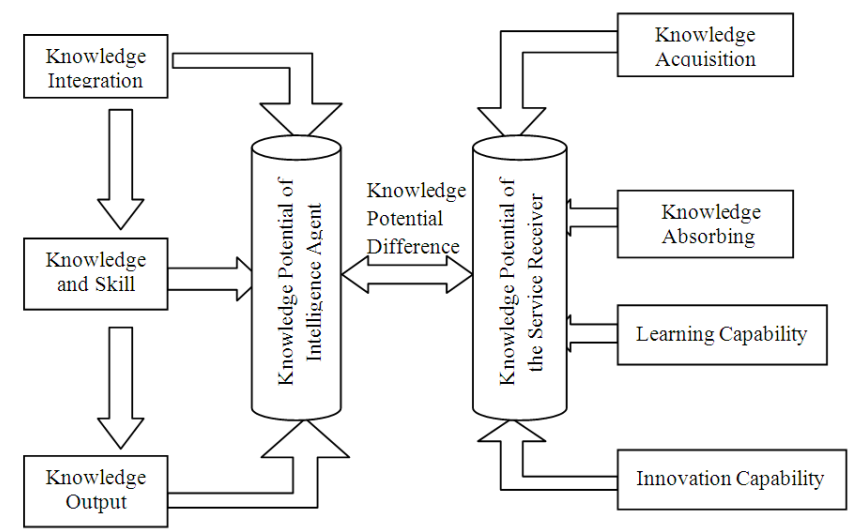

Fig.2. Knowledge Potential Difference Between Intelligence Agent and the Service Receiver

\section{Service Effect Assessment Model}

After receiving the knowledge, the service receiver will digest the knowledge received and coalesce it with the existing knowledge for innovative application.

There is knowledge potential difference in the process of knowledge transfer as the service provider outputs its service to the receiver. In this stage, it is a quantitative change of knowledge from service 
provider to service receiver with less innovation but the quantity of output of service provider and the quantity of input of service receiver determine the smoothness of the service transfer.

Then comes the stage of knowledge appreciation. After receiving the knowledge, the service receiver will digest and absorb the knowledge obtained to coalesce it with the existing knowledge and make innovative application to create new knowledge. In this stage, the knowledge potential gets higher with improved innovation and optimized knowledge structure. In this period, the qualitative change occurs upon the quantitative change and again comes quantitative change. Among them, the learning capability of the receiver plays a crucial role in high efficient transfer that gives an

Important reference to the assessment on the service effects [8]. (See Chart. 3)

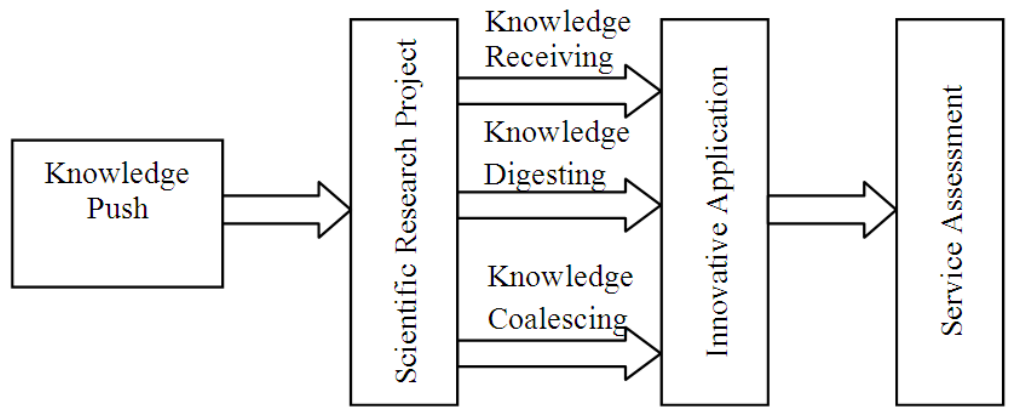

Fig.3.Intelligence Service Assessment Model

It is clear that the service provider has the will and ability of knowledge transfer to support the receiver in solving the problems and it will provide its knowledge output. But influenced by the service circumstances, feedback from the receiver and the capability of knowledge absorbance, digestion and coalescence of the receiver, only a part of the output is absorbed by the receiver. For the progress of the project, however, the receiver should be willing to learn and improve its knowledge potential and the innovation capability.

\section{Conclusion}

Scientific research project oriented intelligence research emphasizes the cooperative interaction between intelligence agent and scientific research personnel and the service circumstances are very important in full knowledge expression and effective transfer. On the one hand, the trust relationship and reciprocal relationship will enforce the communication and feedback, understanding and emotion to improve the service effects; on the other hand, it is of great importance to narrow the gaps between professions, organizations and knowledge potentials of the intelligence agents and scientific researchers for more precise perception of the needs of the project and multi-dimensional expression of the knowledge in order to push knowledge in a quick, accurate and efficient way.

\section{References}

[1] Zhao Li, Zou Manlin, Yang Xi, How to Develop Intelligence Research in a Primary Leveled Scientific Research Institution [J] Informatics Theory and Practice, 2015, 38(1): 27-29.

[2] Xiao Anbang, Considerations on Scientific Information Research Ability Enhancement [J] Informatics Theory and Practice, 2015, 38 (7): 36-38.

[3] Li Xiaosong, Li Bin, A Study on the Growth Model of Scientific Information Agents [J] Informatics Theory and Practice, 2015, 38 (1): 23-26, 34. 
[4] Qin Tiehui, An Enlightenment of Embedability Theory in the Research of Informatics, 2009, 53 (24): 5-6, 20.

[5] Deng Zhonghua, Li Lirui, Lu Yingjun, A Study on Embedded Knowledge Service Basing on the Perception of the Circumstances at the Customer, Vol. A [J] Informatics Theory and Practice, 2014, 37 (9): 16-19, 34.

[6] Deng Zhonghua, Li Lirui, Lu Yingjun, A Study on Embedded Knowledge Service Basing on the Perception of the Circumstances at the Customer, Vol. B [J] Informatics Theory and Practice, 2014, 37 (10): 15-19.

[7] Zhao Junwu, Innovative Methodic System for Scientific Information Research, [J].Aero-engine, 2006, 32 (3) : 53-58.

[8] Li Tianyang, Wang Xin, Quality Control in Intelligence Research---Norm of High Quality Intelligence Research in Rand Corporation [J]. Informatics Theory and Practice, 2012, 35 (12): 126128. 